\title{
Effect of Mineralocorticoid Receptor Antagonists on the Prognosis of Patients with Ventricular Tachyarrhythmias
}

\author{
Tobias Schupp ${ }^{a}$ Max von Zworowsky ${ }^{a} \quad$ Linda Reiser $^{b}$ \\ Mohammad Abumayyaleh $^{a}$ Kathrin Weidner ${ }^{a}$ Kambis Mashayekhi ${ }^{b}$ \\ Thomas Bertsch ${ }^{c}$ Mohammed L. Abba ${ }^{d}$ Ibrahim Akin ${ }^{a}$ Michael Behnes ${ }^{a}$ \\ aDepartment of Cardiology, Angiology, Haemostaseology and Medical Intensive Care, University Medical Centre \\ Mannheim, Medical Faculty Mannheim, Heidelberg University, and European Center for AngioScience (ECAS) and \\ German Center for Cardiovascular Research (DZHK) partner site Heidelberg/Mannheim, Mannheim, Germany; \\ ${ }^{b}$ Department of Cardiology and Angiology II, University Heart Center Freiburg, Bad Krozingen, Germany; ' Institute \\ of Clinical Chemistry, Laboratory Medicine and Transfusion Medicine, Nuremberg General Hospital, Paracelsus \\ Medical University, Nuremberg, Germany; ${ }^{\mathrm{d}}$ Third Department of Medicine, University Medical Centre Mannheim \\ (UMM), Faculty of Medicine Mannheim, University of Heidelberg, Mannheim, Germany
}

\section{Keywords}

Ventricular tachycardia - Ventricular fibrillation · Mortality · Mineralocorticoid receptor antagonists · Spironolactone · Eplerenone $\cdot$ Heart failure

\footnotetext{
Abstract

Introduction: The study sought to assess the effect of treatment with mineralocorticoid receptor antagonists (MRAs) on long-term prognosis of patients with systolic heart failure (HF) surviving index episodes of ventricular tachyarrhythmias. Methods: A large retrospective registry was used including consecutive HF patients with left ventricular ejection fraction $<45 \%$ and index episodes of ventricular tachyarrhythmias from 2002 to 2015 . The primary endpoint was all-cause mortality at 3 years and secondary endpoints were rehospitalization, as well as the composite endpoint consisting of recurrent ventricular tachyarrhythmias, sudden car-
}

diac death and appropriate implantabe cardioverter defibrillator (ICD) therapies at 3 years. Results: 748 patients were included, $20 \%$ treated with MRA and $80 \%$ without. At 3 years, treatment with MRA was not associated with improved allcause mortality ( $22 \%$ vs. $24 \%$, log-rank $p=0.968$; hazard ratio $(\mathrm{HR})=1.008 ; 95 \% \mathrm{Cl} 0.690-1.472 ; p=0.968)$. Accordingly, risk of the composite endpoint ( $28 \%$ vs. $27 \%$; $\mathrm{HR}=1.131 ; 95 \% \mathrm{Cl}$ $0.806-1.589 ; p=0.476)$ and first cardiac rehospitalization (24\% vs. $22 \% ; \mathrm{HR}=1.139 ; 95 \% \mathrm{Cl} 0.788-1.648 ; p=0.489$ ) were not affected by treatment with MRA. Conclusion: In patients with ventricular tachyarrhythmias, treatment with MRA was not associated with improved all-cause mortality at 3 years. The therapeutic effect of MRA treatment in patients with ventricular tachyarrhythmias needs to be reinvestigated within further randomized controlled trials.

(C) 2021 The Author(s)

Published by S. Karger AG, Basel karger@karger.com www.karger.com/pha

Karger $\stackrel{\text { ' }}{5}$

BOPEN ACCESS
(C) 2021 The Author(s)

Published by S. Karger AG, Basel

This article is licensed under the Creative Commons Attribution 4.0 International License (CC BY) (http://www.karger.com/Services OpenAccessLicense). Usage, derivative works and distribution are permitted provided that proper credit is given to the author and the original publisher.
Correspondence to:

Tobias Schupp, t.schupp1902@gmail.com

Max von Zworowsky, max.zworowsky@googlemail.com

Michael Behnes, michael.behnes@umm.de 


\section{Introduction}

Almost 2 decades have passed since the publication of the EPHESUS-trial ("Eplerenone Post - Acute Myocardial Infarction Heart Failure Efficacy and Survival Study") that demonstrated improved survival in patients treated with mineralocorticoid receptor antagonists (MRAs) presenting with systolic heart failure (HF) with severely reduced left ventricular ejection fraction (LVEF $\leq 40 \%)$ following acute myocardial infarction (AMI) [1]. Although improved survival was already seen at 30 days of follow-up, which may be related to reduced cardiac fibrosis in the initial phase of AMI, MRAs are withheld from many patients with systolic HF [2]. This may be related to the rather small amount of randomized controlled trials in the field of MRA treatment in comparison to pharmacological treatment with betablockers or angiotensin-converting enzyme inhibitors (ACEi) or receptor blockers (ARBs) [3-6]. In line, characteristics and treatment strategies for underling cardiac disease (including nationwide health-care supply, improving revascularization strategies, possibility to implant cardiac devices such as an implantable cardioverter defibrillator (ICD), or cardiac resynchronization therapy) demand the need to re-evaluate these pharmacotherapies within the 21th century [7]. Therefore, the therapeutic effect of MRA treatment on the prognosis of todays' patients remains unclear. Even though a reduction of sudden cardiac death (SCD) by $23 \%$ within patients treated with MRA was suggested in HF patients, the benefit of MRA may further decrease in patients with concomitant multi-pharmacological treatment including the guideline-recommended therapies of betablocker and ACEi/ARB [8]. Even less data are available focusing on patients presenting with ventricular tachyarrhythmias [7].

Therefore, this study evaluates the effect of MRA treatment on the primary endpoint of all-cause mortality at 3 years, as well as on secondary endpoints (i.e., composite endpoint of recurrent ventricular tachyarrhythmias, sudden cardiac death and appropriate ICD therapies and cardiac rehospitalization) in HF patients surviving index episodes of ventricular tachyarrhythmias. Furthermore, patients treated with spironolactone were compared to patients treated with eplerenone. Finally, a time trend analysis was performed regarding MRA prescription rates during the study period (i.e., from 2002 to 2015).

\section{Methods}

\section{Data Collection and Documentation}

The present study included retrospectively all consecutive patients surviving index episodes of ventricular tachyarrhythmias (i.e., ventricular tachycardia [VT] or ventricular fibrillation [VF]) on admission from 2002 to 2015 at our institution as recently published [9]. The present study is derived from an analysis of the "Registry of Malignant Arrhythmias and Sudden Cardiac Death - Influence of Diagnostics and Interventions," a single-center registry including consecutive patients presenting with ventricular tachyarrhythmias and aborted cardiac arrest being acutely admitted to the University Medical Center Mannheim, Germany (clinicaltrials.gov identifier: NCT02982473). The study was carried out according to the principles of the Declaration of Helsinki and was approved by the Medical Ethics Committee II of the Medical Faculty Mannheim, the University of Heidelberg, Germany.

\section{Inclusion and Exclusion Criteria}

For the present study, risk stratification was based on the presence or absence of MRA treatment at index hospital discharge. Therefore, patients with in-hospital death were excluded from the present study (Fig. 1; flow-chart). Since current European guidelines recommend MRA treatment in patients with symptomatic $\mathrm{HF}$ and $\mathrm{LVEF}<40 \%$, all patients with preserved LVEF (i.e., $>45 \%$ ) were excluded from the present study [10]. In line, patients with severe hyperkalemia (i.e., serum potassium level $>5.5 \mathrm{mmol} / \mathrm{L}$ ) were excluded from this analysis. The decision to treat patients with MRA was based on the discretion of the cardiologists during routine care according to European guidelines [7, 10-12].

\section{Definition of Case and Control Groups}

The case group (MRA group) comprised all patients with MRA at discharge. Both spironolactone and eplerenone were allowed. The control group (non-MRA group) comprised all patients without treatment with MRA at discharge. All other medical therapies apart from MRA were allowed.

\section{Primary and Secondary Endpoints}

The follow-up period was set at 3 years for all outcomes. The primary prognostic endpoint was all-cause mortality. Secondary endpoints were a composite endpoint (i.e., recurrence of ventricular tachyarrhythmias, sudden cardiac death and appropriate ICD therapies) and first cardiac rehospitalization. Cardiac reshospitalization comprised rehospitalization due to recurrent $\mathrm{VT}$, recurrent VF, AMI, acute heart failure, and inappropriate ICD therapies.

\section{Statistical Methods}

Quantitative data are presented as mean \pm standard error of mean, median and interquartile range, and ranges depending on the distribution of the data and were compared using the Student's $t$ test for normally distributed data or the Mann-Whitney U test for nonparametric data. Deviations from a Gaussian distribution were tested by the Kolmogorov-Smirnov test. Spearman's rank correlation for nonparametric data was used to test univariate correlations. Qualitative data are presented as absolute and relative frequencies and compared using the $\chi^{2}$ test or Fisher's exact test, as appropriate. First, the univariable Kaplan-Meier method was applied to evaluate prognostic differences within the entire cohort. Hazard ratios (HRs) are given together with $95 \%$ confidence intervals.
Schupp et al. 
Fig. 1. Study flowchart. LVEF, left ventricular ejection fraction; MRA, mineralocorticoid receptor antagonists.

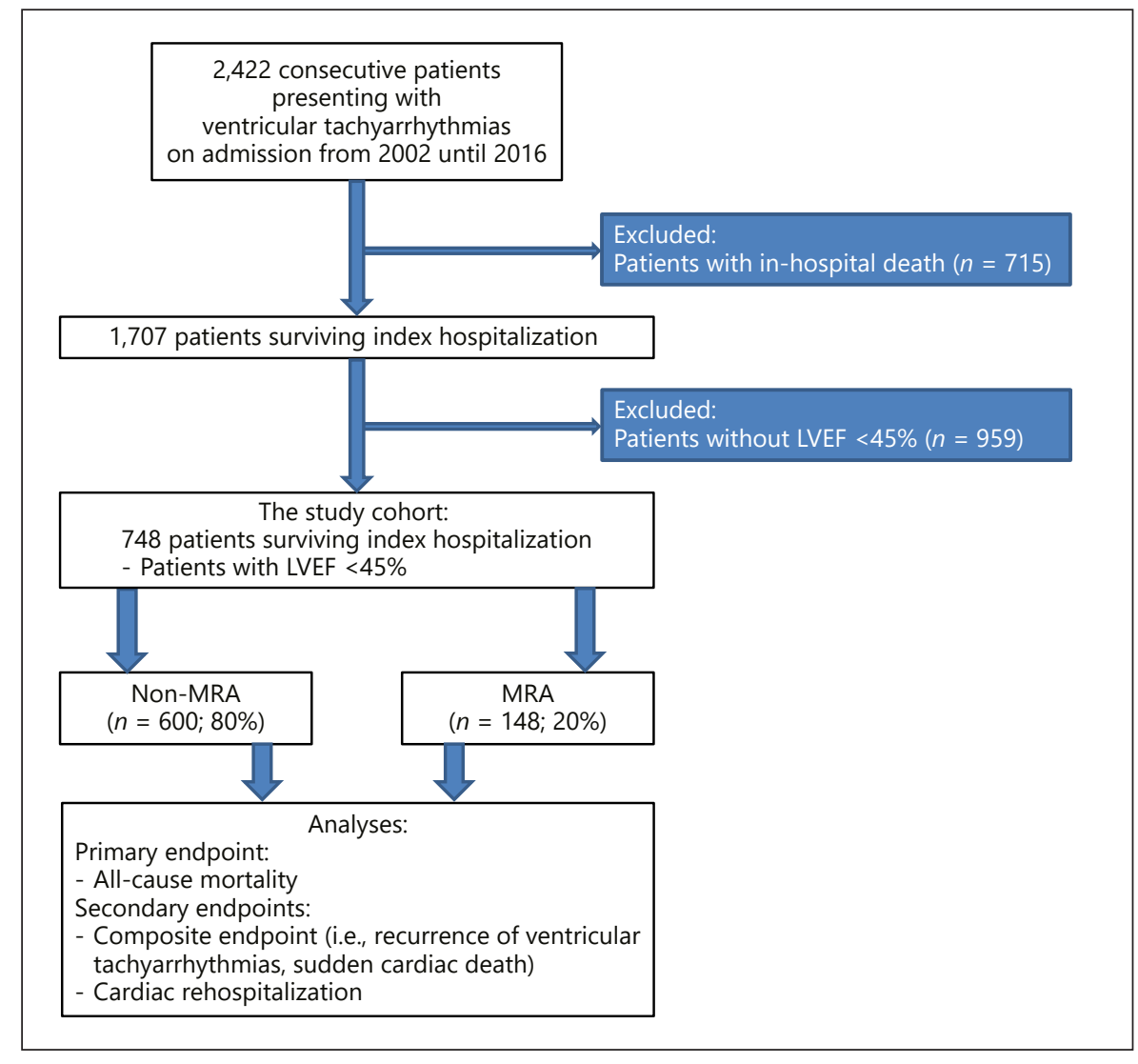

Second, multivariable Cox regression models were developed using the "forward selection" option, where only statistically significant variables $(p<0.05)$ were included and analyzed simultaneously. Predefined variables being used for multivariable Cox-regressions included age, sex, chronic kidney disease, diabetes mellitus, AMI, presence of an ICD, cardiopulmonary resuscitation, coronary artery disease, $\mathrm{LVEF}<35 \%$, and MRA treatment. Cox regression analyses were applied for the endpoint all-cause mortality as well as for secondary endpoints. Thereafter, prognosis of patients treated with spironolactone was compared to patients treated with eplerenone using the Kaplan-Meier method.

Finally, time trend analyses were performed to analyze frequency of MRA prescription during the study period (2002-2015) using the $\chi^{2}$ test. The result of a statistical test was considered significant for $p<0.05$. SAS, release 9.4 (SAS Institute Inc., Cary, NC, USA) and SPSS (version 25, IBM, Armonk, NY, USA) were used for statistics.

\section{Results}

\section{Study Population}

From 2002 to 2015, a total of 1,707 patients survived index episodes of ventricular tachyarryhtmias. Of those, 954 patients were not eligible for MRA therapy due to preserved LVEF (i.e., LVEF >45\%) and 5 patients for hyperkalaemia with serum potassium levels $>5.5 \mathrm{mmol} / \mathrm{L}$ (Fig. 1; flowchart). The final study cohort comprised 748 consecutive patients with systolic HF (i.e., LVEF $<45 \%$ ). All patients survived an index episode of ventricular tachyarrhythmias and were discharged with or without MRA (non-MRA $80 \%$ vs. MRA 20\%; $p=0.001$ ). Target dosages of MRA were reached already at discharge, as seen for spironolactone in $60 \%$ (mean dosage $34 \mathrm{mg}$ per day) and for eplerenone in $40 \%$ (mean dosage $27 \mathrm{mg}$ per day) (Table 1).

As seen in Table 1, the median age was 66 years, and most patients were males in both subgroups (79-80\%; $p=0.743)$. Types of index ventricular tachyarrhythmias were similar in MRA and non-MRA patients (i.e., VT: 72-74\%; VF: $26-28 \%$; $p=0.718$ ). A history of HF was more common in patients with MRA treatment (53\% vs. $40 \% ; p=0.003$ ). Cardiovascular risk factors (i.e., arterial hypertension ( $66 \%$ vs. $63 \% ; p=0.401)$, diabetes mellitus ( $29 \%$ vs. $30 \%$; $p=0.853$ ), hyperlipidaemia ( $33 \%$ vs. $39 \%$; $p=0.211)$, smoking ( $32 \%$ vs. $31 \% ; p=0.890)$, and cardiac family history ( $14 \%$ vs. $12 \%$; $p=0.368)$ were equally distributed in both groups. Accordingly, rates of chronic 
Table 1. Baseline characteristics

\begin{tabular}{|c|c|c|c|}
\hline Characteristic & $\begin{array}{l}\text { Non-MRA } \\
(n=600 ; 80 \%)\end{array}$ & $\begin{array}{l}\text { MRA } \\
(n=148 ; 20 \%)\end{array}$ & $p$ value \\
\hline \multicolumn{4}{|l|}{ Study drugs } \\
\hline Spironolactone, $n(\%)$ & - & $89(60)$ & - \\
\hline Mean \pm SEM, mg/day & - & $34 \pm 1.9$ & - \\
\hline Eplerenone, $n(\%)$ & - & $59(40)$ & - \\
\hline Mean \pm SEM, mg/day & - & $27 \pm 1.6$ & - \\
\hline Age, median (range) & $66(15-90)$ & $65(27-83)$ & 0.070 \\
\hline Male gender, $n(\%)$ & $471(79)$ & $118(80)$ & 0.743 \\
\hline \multicolumn{4}{|c|}{ Ventricular tachyarrhythmias at index, $n(\%)$} \\
\hline VT & $433(72)$ & $109(74)$ & 0.718 \\
\hline VF & $167(28)$ & $39(26)$ & \\
\hline \multicolumn{4}{|l|}{ Cardiovascular risk factors, $n(\%)$} \\
\hline Arterial hypertension & $375(63)$ & $98(66)$ & 0.401 \\
\hline Diabetes mellitus & $179(30)$ & $43(29)$ & 0.853 \\
\hline Hyperlipidemia & $232(39)$ & $49(33)$ & 0.211 \\
\hline Smoking & $187(31)$ & $47(32)$ & 0.890 \\
\hline Cardiac family history & $69(12)$ & $21(14)$ & 0.368 \\
\hline \multicolumn{4}{|l|}{ Comorbidities at index stay, $n(\%)$} \\
\hline Prior myocardial infarction & $229(38)$ & $54(37)$ & 0.706 \\
\hline Prior coronary artery disease & $353(59)$ & $80(54)$ & 0.292 \\
\hline Prior heart failure & $240(40)$ & $79(53)$ & 0.003 \\
\hline Atrial fibrillation & $211(35)$ & $66(45)$ & 0.033 \\
\hline Nonischemic cardiomyopathy & $80(13)$ & $29(20)$ & 0.053 \\
\hline Cardiopulmonary resuscitation & $151(25)$ & $26(18)$ & 0.120 \\
\hline In hospital & $52(9)$ & $7(5)$ & 0.075 \\
\hline Out of hospital & $99(17)$ & $19(13)$ & 0.261 \\
\hline Chronic kidney disease & $280(48)$ & $71(48)$ & 0.869 \\
\hline COPD & $52(9)$ & $19(13)$ & 0.121 \\
\hline Stroke & $14(2)$ & $6(4)$ & 0.245 \\
\hline Intracranial hemorrhage & $2(0.3)$ & $1(0.7)$ & 0.484 \\
\hline Coronary angiography, $n$ (\%) & $411(69)$ & $83(56)$ & 0.004 \\
\hline No evidence of CAD & $86(21)$ & $22(27)$ & 0.663 \\
\hline 1-Vessel disease & $90(22)$ & $18(22)$ & \\
\hline 2-Vessel disease & $94(23)$ & $19(23)$ & \\
\hline 3-Vessel disease & $141(34)$ & $24(29)$ & \\
\hline Chronic total occlusion & $108(26)$ & $23(28)$ & 0.787 \\
\hline Presence of CABG & $92(22)$ & $14(17)$ & 0.264 \\
\hline $\mathrm{PCl}$ & $145(35)$ & $29(35)$ & 0.953 \\
\hline AMI & $130(22)$ & $27(18)$ & 0.360 \\
\hline STEMI & $44(7)$ & $12(8)$ & 0.748 \\
\hline NSTEMI & $86(14)$ & $15(10)$ & 0.181 \\
\hline Hyperkalemia & $4(1)$ & $1(1)$ & 1.000 \\
\hline Hypokalemia & $25(4)$ & $18(12)$ & 0.001 \\
\hline \multicolumn{4}{|l|}{ LVEF, $n(\%)$} \\
\hline $44-35 \%$ & $228(38)$ & $38(26)$ & 0.005 \\
\hline$<35 \%$ & $372(62)$ & $110(74)$ & \\
\hline \multicolumn{4}{|l|}{ Cardiac therapies at index, $n(\%)$} \\
\hline Electrophysiological examination & $189(32)$ & $38(26)$ & 0.167 \\
\hline VT ablation therapy & $39(7)$ & $15(10)$ & 0.126 \\
\hline Presence of an ICD, $n(\%)$ & $400(67)$ & $118(80)$ & 0.002 \\
\hline ICD & $365(91)$ & $101(86)$ & 0.277 \\
\hline$s-I C D$ & $1(0.1)$ & $2(0.2)$ & \\
\hline CRT-D & $34(9)$ & $15(13)$ & \\
\hline
\end{tabular}


Table 1 (continued)

\begin{tabular}{llll}
\hline Characteristic & $\begin{array}{l}\text { Non-MRA } \\
(n=600 ; 80 \%)\end{array}$ & $\begin{array}{l}\text { MRA } \\
(n=148 ; 20 \%)\end{array}$ & $p$ value \\
\hline $\begin{array}{l}\text { Medication at discharge, } n(\%) \\
\text { Beta-blocker }\end{array}$ & & & \\
ACE-inhibitor & $525(88)$ & $133(90)$ & 0.428 \\
ARB & $419(70)$ & $107(72)$ & 0.557 \\
Statin & $68(12)$ & $26(18)$ & $\mathbf{0 . 0 4 0}$ \\
Amiodarone & $407(68)$ & $99(67)$ & 0.826 \\
Digitalis & $135(23)$ & $41(28)$ & 0.181 \\
\hline
\end{tabular}

Bold type indicates $p<0.05$. ACE, angiotensin converting enzyme; $A R B$, angiotensin receptor blocker; $C A B G$, coronary artery bypass grafting; $C A D$, coronary artery disease; COPD, chronic obstructive pulmonary disease; CRT-D, cardiac resynchronization therapy with defibrillator; (s-)ICD, (subcutaneous) implantable cardioverter defibrillator; LVEF, left ventricular ejection fraction; NSTEMI, non-ST-segment myocardial infarction; $\mathrm{PCl}$, percutaneous coronary intervention; SEM, standard error of mean; STEMI, ST-segment MI; VT, ventricular tachycardia; VF, ventricular fibrillation; MRA, mineralocorticoid receptor antagonist; AMI, acute myocardial infarction.

Table 2. Primary and secondary endpoints, follow-up data

\begin{tabular}{|c|c|c|c|}
\hline Characteristics & Non-MRA $(n=600 ; 80 \%)$ & MRA $(n=148 ; 20 \%)$ & $p$ value \\
\hline \multicolumn{4}{|l|}{ Primary endpoint, $n(\%)$} \\
\hline All cause-mortality, at 3 years & $142(24)$ & $33(22)$ & 0.725 \\
\hline Rehospitalization, at 3 years & $130(22)$ & $36(24)$ & 0.486 \\
\hline Composite endpoint (recurrent ventricular tachyarrhythmias; sudden & & & \\
\hline Hospitalization time, days (median (IQR)) & $13(8-23)$ & $12(8-24)$ & 1.000 \\
\hline ICU time, days (median (IQR)) & $3(0-8)$ & $3(0-7)$ & 0.931 \\
\hline Survival time, days (mean; median (range)) & 1,$888 ; 1,682(690-2,973)$ & 1,$350 ; 1,121(416-2,118)$ & 0.001 \\
\hline
\end{tabular}

Level of significance $p \leq 0.05$. Bold type indicates $p \leq 0.05$. ICU, invasive care unit; IQR, interquartile range; MRA, mineralocorticoid receptor antagonist.

kidney disease ( $48 \%$ vs. $48 \%$; $p=0.869)$, AMI ( $18 \%$ vs. $20 \% ; p=0.36)$, and cardiopulmonary resuscitation $(18 \%$ vs. $25 \%$; $p=0.12$ ) did not significantly differ among patients with and without MRA therapy. A higher rate of atrial fibrillation was observed in patients on MRA treatment $(45 \%$ vs. $35 \% ; p=0.033)$, which was mainly attributed to higher rates of paroxysmal atrial fibrillation. In line, $\mathrm{LVEF}<35 \%$ was more common in patients on MRA treatment than in patients without $(74 \%$ vs. $62 \%$; $p=$ $0.005)$, alongside with a higher rate of patients discharged with an ICD ( $80 \%$ vs. $67 \% ; p=0.002)$. A transvenous ICD was mostly implanted device in patients with or without MRA ( $86 \%$ vs. $91 \%$; $p=0.277)$. Despite a slightly higher rate of concomitant pharmacological treatment with
ARB ( $18 \%$ vs. $12 \%$; $p=0.040)$ and digitalis ( $27 \%$ vs. $19 \%$; $p=0.031$ ) was seen in the MRA group, whereas no further significant differences were observed in between both groups (Table 1).

\section{Primary and Secondary Endpoints}

Median follow-up within the study cohort was 3.1 years (interquartile range $1.1-5.8$ years). At 3 years of follow-up, the primary endpoint of all-cause mortality occurred in $22 \%$ of patients with MRA and $25 \%$ of patients without (log-rank $p=0.968$ ) (Table 2; Fig. 2, left panel). Accordingly, risk of all-cause mortality was not affected by MRA treatment ( $\mathrm{HR}=1.008$; $95 \%$ CI 0.690 $1.472 ; p=0.968)$. In line, no statistical differences were 


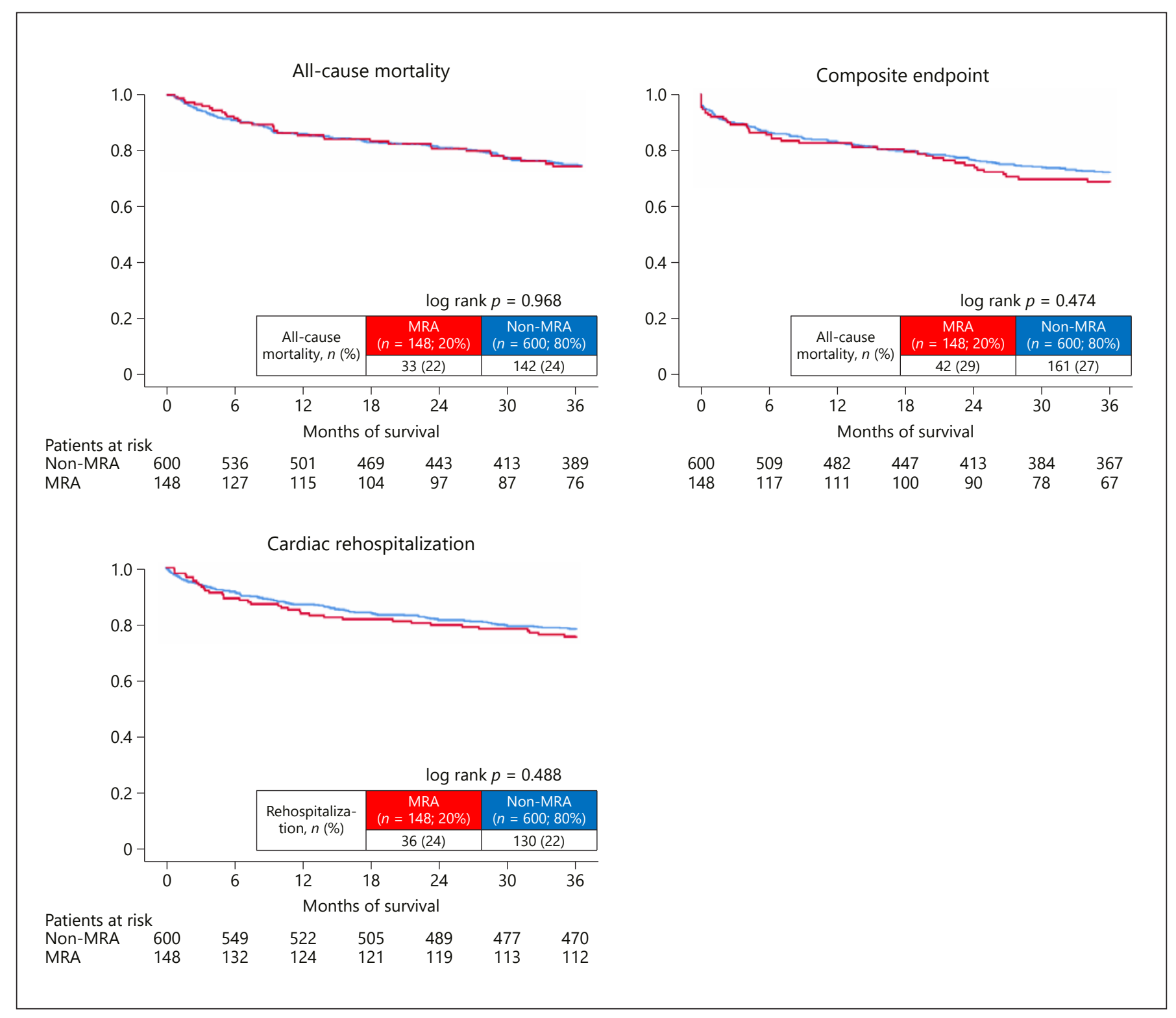

Fig. 2. Effect of MRA treatment on all-cause mortality (left panel), the composite endpoint (middle panel), and cardiac rehospitalization (right panel) at 3 years. MRA, mineralocorticoid receptor antagonist.

observed regarding the risk of the composite endpoint ( $28 \%$ vs. $27 \%$; $\mathrm{HR}=1.131 ; 95 \%$ CI $0.806-1.589 ; p=0.476)$, as well as the risk of first cardiac rehospitalization $(24 \%$ vs. $22 \%$; HR $=1.139 ; 95 \%$ CI $0.788-1.648 ; p=0.489$ ) (Fig. 2, middle and right panels).

\section{Multivariable Cox Regression Models}

After multivariable adjustment, MRA treatment was not associated with the risk of all-cause mortality in patients with ventricular tachyarrhythmias $(\mathrm{HR}=1.174$; 95\% CI 0.794-1.736; $p=0.421$ ) (Table 3). Accordingly,
MRA patients were not associated with the risk of the composite endpoint $(\mathrm{HR}=0.938$; 95\% CI 0.665-1.323; $p=0.714)$ and cardiac rehospitalization $(\mathrm{HR}=1.061$; 95\% CI $0.730-1.543 ; p=0.756)$. Especially increasing age $(\mathrm{HR}=1.029)$, diabetes mellitus $(\mathrm{HR}=1.749)$, chronic kidney disease $(\mathrm{HR}=1.464)$, and cardiopulmonary resuscitation $(\mathrm{HR}=1.281)$ were associated with impaired long-term survival. In contrast, improved survival was observed in patients with an ICD $(\mathrm{HR}=0.385)$ (Table 3).
40

Pharmacology 2022;107:35-45 DOI: $10.1159 / 000520310$
Schupp et al. 
Comparison of Spironolactone versus Eplerenone

Thereafter, the prognostic impact of spironolactone was directly compared to eplerenone, whereas both types of MRA were associated with similar risk of allcause mortality $(23 \%$ vs. $22 \%$; $p=0.991$; $\mathrm{HR}=1.004$; $95 \%$ CI $0.499-2.019 ; p=0.991)$, the composite endpoint (32\% vs. $24 \% ; p=0.344 ; \mathrm{HR}=1.360 ; 95 \%$ CI $0.716-$ $2.584 ; p=0.347)$, and cardiac rehospitalization ( $25 \%$ vs. $24 \% ; p=0.973 ; \mathrm{HR}=0.988 ; 95 \%$ CI $0.506-1.932 ; p=$ 0.973) (Fig. 3).

\section{Time Trend Analysis}

Finally, we investigated a time trend analysis regarding the prescription of MRA during the study period. From 2002 to 2015, treatment with MRA has significantly increased from $13.8 \%$ in 2002 to $45.2 \%$ in 2015 ( $p=0.001$ for the trend) (Fig. 4).

\section{Discussion}

The present study evaluates the effect of MRA treatment on the primary endpoint of all-cause mortality, as well as on secondary endpoints, such as a composite endpoint (i.e., recurrence of ventricular tachyarrhythmias and sudden cardiac death) and cardiac rehospitalization in $\mathrm{HF}$ patients with LVEF $<45 \%$ surviving index episodes of ventricular tachyarrhythmias. In this cohort of HF patients with ventricular tachyarrhythmias, only $20 \%$ of patients were treated with MRA at hospital discharge; however, treatment rates with MRA have significantly increased from $13.8 \%$ to $45.2 \%$ during the study period. These data suggest no prognostic impact of MRA therapy on long-term prognosis in patients with ventricular tachyarrhythmias. Finally, prognosis of patients treated with spironolactone did not differ from that of those treated with eplerenone.

Despite the class IA indication in European guidelines for chronic HF patients [10], treatment with MRA is often withheld from a large group of HF patients [13]. This may be related to the rather small number of randomized studies investigating the potential role of MRA in comparison to other HF pharmacotherapies (such as betablockers or ACEi), an overestimation of potential side effects (i.e., hyperkalaemia and arterial hypotension with low-output resulting in impaired renal function), and the strategy to initiate MRA treatment after up-titration of concomitant beta-blocker and ACEi/ARB treatment [14] . Subsequently, MRA treatment was applied in only $67 \%$ of eligible patients within the ESC Heart Failure Long-Term
Table 3. Multivariable Cox regression analyses

\begin{tabular}{|c|c|c|c|}
\hline Endpoint & $\mathrm{HR}$ & $95 \% \mathrm{Cl}$ & $p$ value \\
\hline \multicolumn{4}{|l|}{ Mortality } \\
\hline Age & 1.029 & $1.013-1.045$ & 0.001 \\
\hline Gender & 1.335 & $0.904-1.971$ & 0.146 \\
\hline Diabetes & 1.749 & $1.290-2.372$ & 0.001 \\
\hline Chronic kidney disease & 1.464 & $1.074-1.995$ & 0.016 \\
\hline $\mathrm{AMI}$ & 0.544 & $0.348-0.849$ & 0.007 \\
\hline KHK Kombi & 1.285 & $0.875-1.888$ & 0.202 \\
\hline LVEF $<35 \%$ & 1.859 & $1.305-2.648$ & 0.001 \\
\hline CPR & 1.281 & $1.015-1.616$ & 0.037 \\
\hline Presence of ICD & 0.385 & $0.279-0.532$ & 0.001 \\
\hline MRA & 1.174 & $0.794-1.736$ & 0.421 \\
\hline \multicolumn{4}{|l|}{ Composite endpoint } \\
\hline Age & 1.007 & $0.995-1.020$ & 0.267 \\
\hline Gender & 1.115 & $0.768-1.616$ & 0.568 \\
\hline Diabetes & 0.782 & $0.568-1.077$ & 0.132 \\
\hline Chronic kidney disease & 0.944 & $0.710-1.255$ & 0.690 \\
\hline AMI & 0.956 & $0.610-1.497$ & 0.843 \\
\hline KHK Kombi & 0.778 & $0.561-1.079$ & 0.132 \\
\hline LVEF <35\% & 1.027 & $0.755-1.396$ & 0.867 \\
\hline CPR & 0.733 & $0.542-0.991$ & 0.043 \\
\hline Presence of ICD & 4.628 & $2.806-7.634$ & 0.001 \\
\hline MRA & 0.938 & $0.665-1.323$ & 0.714 \\
\hline \multicolumn{4}{|l|}{ Rehospitalization } \\
\hline Age & 1.014 & $0.999-1.029$ & 0.065 \\
\hline Gender & 0.937 & $0.631-1.389$ & 0.745 \\
\hline Diabetes & 0.995 & $0.711-1.393$ & 0.979 \\
\hline Chronic kidney disease & 1.221 & $0.890-1.675$ & 0.217 \\
\hline $\mathrm{AMI}$ & 1.713 & $1.126-2.608$ & 0.012 \\
\hline KHK Kombi & 1.145 & $0.761-1.723$ & 0.516 \\
\hline LVEF $<35 \%$ & 0.890 & $0.638-1.240$ & 0.490 \\
\hline CPR & 0.756 & $0.552-1.036$ & 0.082 \\
\hline Presence of ICD & 3.343 & $2.100-5.320$ & 0.001 \\
\hline MRA & 1.061 & $0.730-1.543$ & 0.756 \\
\hline
\end{tabular}

Level of significance $p \leq 0.05$. Bold type indicates statistical significance. AMl; acute myocardial infarction; $\mathrm{Cl}$; confidence interval; CPR; cardiopulmonary resuscitation; HR; hazard ratio; ICD; implantable cardioverter-defibrillator; LVEF, left ventricular ejection faction; MRA; mineralocorticoid receptor antagonist.

Registry, whereas at least $92 \%$ of the patients were treated with beta -blockers and ACEi/ARB, respectively [15]. In line, only $22 \%$ of MRA eligible patients were treated with MRA within a recently published study by Wong et al. [2], including 317 patients with AMI with systolic HF. In line, in $<10 \%$ of all patients without MRA treatment at index hospital discharge, MRA was initiated with the following 3 months $[2,16]$. However, endpoint-related data were not provided within the study by Wong et al. [2]. Thus, rather low prescription rates of MRA (i.e., only $20 \%$ of MRA eligible patients) in the present study underline the 


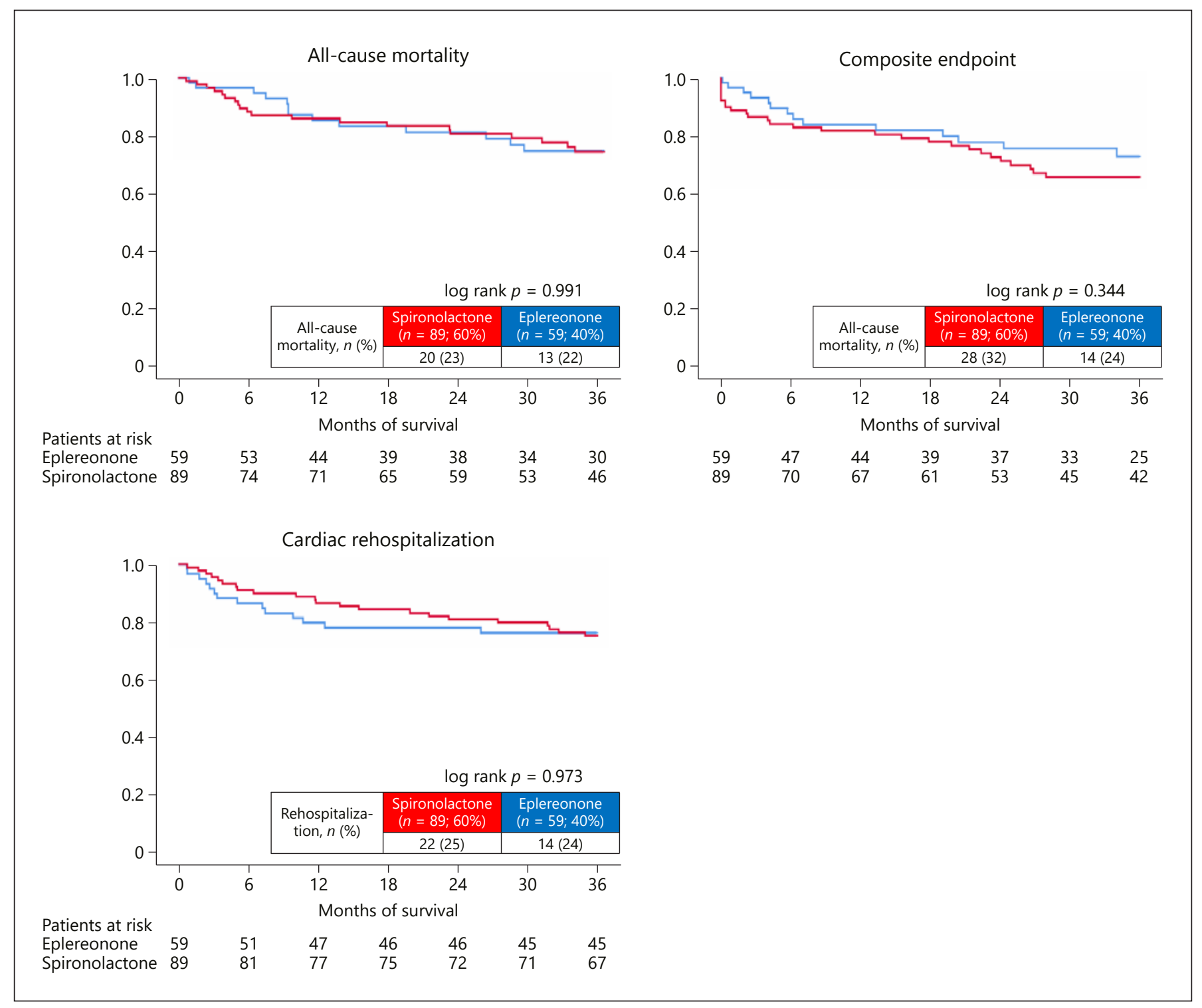

Fig. 3. Effect of spironolactone compared to eplerenone treatment regarding all-cause mortality (left panel), as well as the composite endpoint (middle panel) and cardiac rehospitalization (right panel).

need to further improve guideline adherence for MRA treatment, although a significant trend to improvement to guideline adherence was observed. Of note, this trend may also explain the shorter follow-up duration of MRA patients within the present study, since a large group of MRA patients were included at the end of the study period.

Besides investigating the association of MRA treatment and ventricular tachyarrhythmias, many studies focus on the potential role of MRA on atrial fibrillation [17]. A recently published meta-analysis including 5 studies ( 3 randomized trials and 2 observational studies) and 1,337 patients demonstrated $31 \%$ lower risk of atrial fibrillation in patients treated with MRA as compared to patients without. In contrast, the present study did not identify MRA to improve survival and reduce the risk of the composite arrhythmic endpoint. Moreover, the meta-analysis by Liu et al. [17] investigated the prognostic value of spironolactone compared to eplerenone, demonstrating decreased risk of atrial fibrillation in patients treated with spironolactone only. However, in patients with ventricular tachyarrhythmias on admission, pa-
42

Pharmacology 2022;107:35-45 DOI: $10.1159 / 000520310$
Schupp et al. 
Fig. 4. Time trend analysis regarding prescription rates of MRA therapy during the study period (2002-2015).

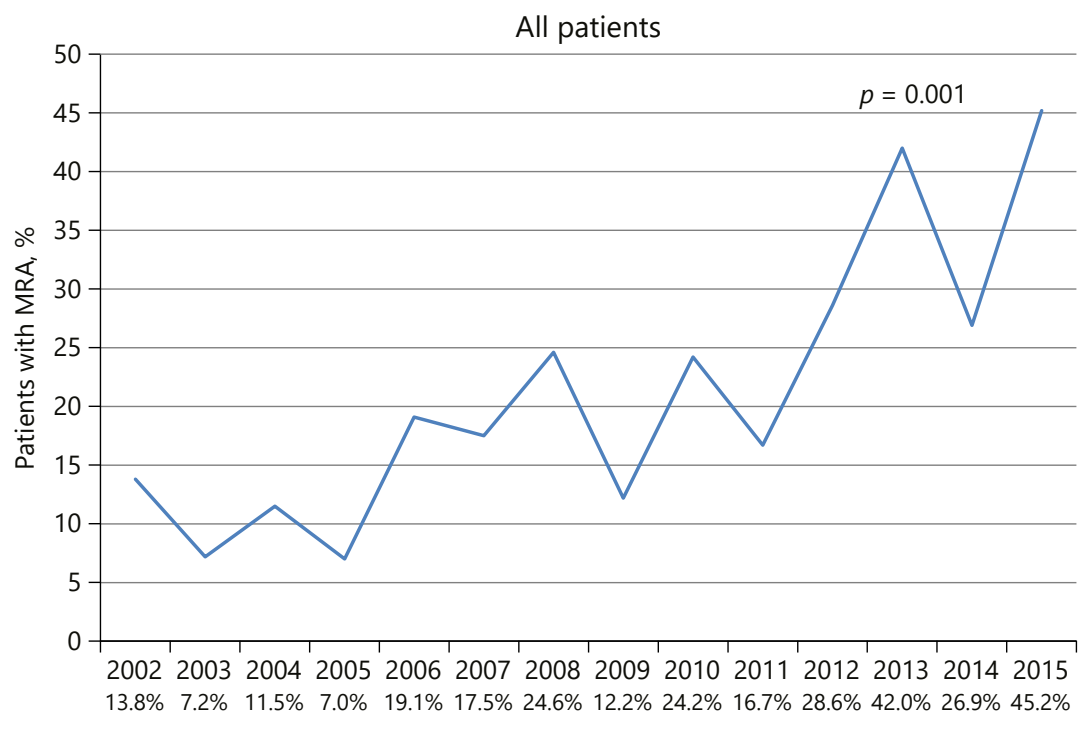

tients treated with spironolactone and eplerenone were associated with comparable long-term outcomes in the present study.

In line, a recently published study by Hasin et al. [18] investigated the prognostic value of HF therapies in 186 HF patients with primary preventive ICD or cardiac resynchronization therapy-D devices. Thus, it was demonstrated that treatment with beta-blockers or ACEi/ $A R B$, or MRA treatment did not affect the risk of ventricular tachyarrhythmias. This study confirms the findings by Hasin et al. [18] for "secondary prevention" of sudden cardiac death, suggesting no additional impact of MRA treatment on the combined arrhythmic endpoint. These findings are in line with the randomized SPIRIT trial that demonstrated no prognostic impact of spironolactone ( $25 \mathrm{mg}$ daily) on the incidence of VT/VF, as well as on appropriate ICD therapies within 90 ICD recipients at moderate to high risk for VT/VF [19].

Even though the present study did not identify MRA treatment associated with improved long-term outcomes in this "all-comers" study, there may be some subgroups of patients where MRA treatment could be more effective. In line, a recently published study by Cohen et al. [20] investigated the prognosis of specific HF phenotypes, as well as the prognostic value of MRA treatment in these patients, including over 3,000 patients from the "TOPCAT" (Treatment of Preserved Cardiac Function Heart Failure with an Aldosterone
Antagonist Trial) study. Thus, especially obese patients suffering from diabetes mellitus showed better response to MRA treatment than other phenotypes [20]. Of note, this may especially be related to the so-called diabetic cardiomyopathy [21]. Diabetic cardiomyopathy is related to reactive oxygen species, inflammation, and activation of the renin-angiotensin-aldosterone system, leading to functional myocardial changes and subsequently to left ventricular hypertrophy and adverse remodeling. The prognostic benefit of eplerenone treatment for diabetic cardiomyopathy was already investigated within various studies, suggesting improved long-term outcomes, but increased risk of hyperkalemia in patients treated with MRA $[22,23]$. It will therefore be of major interest to re-evaluate the impact of MRA treatment in patients with ventricular tachyarrhythmias, as well as on arrhythmic endpoints, within prespecified subgroups (such as patients with diabetic cardiomyopathy) to identify the subgroup of patients that benefit from MRA treatment. In conclusion, rather low subscription rates of MRA (20\%) were observed in the present study including consecutive patients with HF and ventricular tachyarrhythmias, which have increased from $13.8 \%$ to $45.2 \%$ during the study period. It was demonstrated that MRAs were not associated with long-term prognostic endpoints (i.e., all-cause mortality, composite endpoint and cardiac rehospitalization) at 3 years. Finally, prognosis of patients treated with spironolactone or eplerenone was comparable. 


\section{Study Limitations}

This observational and retrospective registry-based analysis reflects a realistic picture of consecutive healthcare supply of high-risk patients presenting with ventricular tachyarrhythmias. Lost to follow-up rate regarding the evaluated endpoint of all-cause mortality was minimal. All available clinical data as outlined in the methods were documented reliably by individual cardiologists during routine clinical care being blinded to final analyses relativized the use of an independent clinical event committee. Pharmacological therapies were based on discharge medication at index event only and based on the decision of the treating physicians during routine clinical care. Therefore, data regarding discontinuation and new initiation of MRA at follow-up, the potential decreasing treatment effect related to MRA in the presence of administered polypharmacy, HF symptoms, biomarkers such as B-type natriuretic peptide (BNP), rehospitalization at other medical centres, as well as the prognostic impact of MRA on changes of LVEF during follow-up were not available due to the retrospective design and therefore beyond the scope of the present study.

\section{Statement of Ethics}

The study was approved by the Medical Ethics Committee II of the Medical Faculty Mannheim, University of Heidelberg, Germany (Ethical Approval Number: 2016-612N-MA). In accordance with local guidelines, consent to participate was not necessary because of the retrospective study design.

\section{Conflict of Interest Statement}

The authors declare that they do not have any conflict of interest.

\section{Funding Sources}

This manuscript did not receive any funding.

\section{Author Contributions}

T.S. substantially contributed to the conception and design of the work, data acquisition and analysis, as well as interpretation of data for the work, and drafted the work and revisited for critically important intellectual content. M.Z., L.R., M.A., K.W., M.A., K.M., and T.B. substantially contributed to analysis as well as interpretation of data for the work, and drafted the work and revisited for critically important intellectual content. I.A. and M.B. substantially contributed to the conception and design of the work, data acquisition and analysis, as well as interpretation of data for the work, and drafted the work and revisited for critically important intellectual content.

\section{Data Availability Statement}

The data that support the findings of this study are available on request from the corresponding author. The data are not publicly available due their containing information that could compromise the privacy of research participants.

\section{References}

1 Pitt B, Remme W, Zannad F, Neaton J, Martinez F, Roniker B, et al. Eplerenone, a selective aldosterone blocker, in patients with left ventricular dysfunction after myocardial infarction. N Engl J Med. 2003;348(14):130921.

2 Wong E, Fordyce C, Wong G, Lee T, PerryArnesen M, Mackay M, et al. Predictors of the use of mineralocorticoid receptor antagonists in patients with left ventricular dysfunction post-st-segment elevation myocardial infarction. J Am Heart Assoc. 2021.

3 Effect of metoprolol CR/XL in chronic heart failure: metoprolol CR/XL randomised intervention trial in congestive heart failure (MERIT-HF). Lancet. 1999;353(9169):2001-7.

4 The cardiac insufficiency bisoprolol study II (CIBIS-II): a randomised trial. Lancet. 1999; 353(9146):9-13.

5 Køber L, Torp-Pedersen C, Carlsen JE, Bagger $\mathrm{H}$, Eliasen P, Lyngborg K, et al. A clinical trial of the angiotensin-converting-enzyme inhibitor trandolapril in patients with left ventricular dysfunction after myocardial infarction. Trandolapril cardiac evaluation (TRACE) study group. N Engl J Med. 1995;333(25):1670-6.

6 Pfeffer MA, Braunwald E, Moyé LA, Basta L, Brown EJ Jr, Cuddy TE, et al. Effect of captopril on mortality and morbidity in patients with left ventricular dysfunction after myocardial infarction. Results of the survival and ventricular enlargement trial. The SAVE Investigators. N Engl J Med. 1992;327(10):66977.

7 Priori SG, Blomstrom-Lundqvist C, Mazzanti A, Blom N, Borggrefe M, Camm J, et al. 2015 ESC guidelines for the management of patients with ventricular arrhythmias and the prevention of sudden cardiac death: the task force for the management of patients with ventricular arrhythmias and the prevention of sudden cardiac death of the European society of cardiology (ESC). Endorsed by: association for European paediatric and congenital cardiology (AEPC). Eur Heart J. 2015;36(41): 2793-867.
8 Rossello X, Ariti C, Pocock SJ, Ferreira JP, Girerd N, McMurray JJV, et al. Impact of mineralocorticoid receptor antagonists on the risk of sudden cardiac death in patients with heart failure and left-ventricular systolic dysfunction: an individual patient-level metaanalysis of three randomized-controlled trials. Clin Res Cardiol. 2019;108(5):477-86.

9 Schupp T, Behnes M, Weiß C, Nienaber C, Lang S, Reiser L, et al. Beta-blockers and ace inhibitors are associated with improved survival secondary to ventricular tachyarrhythmia. Cardiovasc Drugs Ther. 2018;32(4):353-63.

10 Ponikowski P, Voors AA, Anker SD, Bueno $\mathrm{H}$, Cleland JG, Coats AJ, et al. ESC guidelines for the diagnosis and treatment of acute and chronic heart failure: the task force for the diagnosis and treatment of acute and chronic heart failure of the European society of cardiology (ESC) developed with the special contribution of the heart failure association (HFA) of the ESC. Eur Heart J. 2016;37(27): 2129-200. 
11 Kirchhof P, Benussi S, Kotecha D, Ahlsson A, Atar D, Casadei B, et al. 2016 ESC guidelines for the management of atrial fibrillation developed in collaboration with EACTS. Europace. 2016;18(38):1609-78.

12 Al-Khatib SM, Stevenson WG, Ackerman MJ, Bryant WJ, Callans DJ, Curtis AB, et al. 2017 AHA/ACC/HRS guideline for management of patients with ventricular arrhythmias and the prevention of sudden cardiac death. A report of the American college of cardiology/ American heart association task force on clinical practice guidelines and the heart rhythm society. Circulation. 2018 Sep 25;138(13): e210.

13 Duran JM, Gad S, Brann A, Greenberg B. Mineralocorticoid receptor antagonist use following heart failure hospitalization. ESC Heart Fail. 2020;7(2):482-92.

14 Ferreira JP, Rossignol P, Machu JL, Sharma A, Girerd N, Anker SD, et al. Mineralocorticoid receptor antagonist pattern of use in heart failure with reduced ejection fraction: findings from BIOSTAT-CHF. Eur J Heart Fail. 2017;19(10):1284-93.
15 Maggioni AP, Anker SD, Dahlström U, Filippatos G, Ponikowski P, Zannad F, et al. Are hospitalized or ambulatory patients with heart failure treated in accordance with European Society of Cardiology guidelines? Evidence from 12,440 patients of the ESC heart failure long-term registry. Eur J Heart Fail. 2013;15(10):1173-84.

16 Schupp T, Akin I, Behnes M. Pharmacological treatment following myocardial infarction: how large is the gap between guideline recommendations and routine clinical care? J Am Heart Assoc. 2021;10(14):e021799.

17 Liu T, Korantzopoulos P, Shao Q, Zhang Z, Letsas KP, Li G. Mineralocorticoid receptor antagonists and atrial fibrillation: a metaanalysis. Europace. 2016;18(5):672-8.

18 Hasin T, Davarashvili I, Michowitz Y, Farkash R, Presman H, Glikson M, et al. Association of guideline-based medical therapy with malignant arrhythmias and mortality among heart failure patients implanted with cardioverter defibrillator (ICD) or cardiac resynchronization-defibrillator device (CRTD). J Clin Med. 2021;10(8):1753.
19 Zarraga IG, Dougherty CM, MacMurdy KS, Raitt MH. The effect of spironolactone on ventricular tachyarrhythmias in patients with implantable cardioverter-defibrillators. Circ Arrhythm Electrophysiol. 2012;5(4):739-47.

20 Cohen JB, Schrauben SJ, Zhao L, Basso MD, Cvijic ME, Li Z, et al. Clinical phenogroups in heart failure with preserved ejection fraction: detailed phenotypes, prognosis, and response to spironolactone. JACC Heart Fail. 2020; 8(3):172-84

21 Dannenberg L, Weske S, Kelm M, Levkau B, Polzin A. Cellular mechanisms and recommended drug-based therapeutic options in diabetic cardiomyopathy. Pharmacol Ther. 2021;228:107920.

22 Chen MD, Dong SS, Cai NY, Fan MD, Gu SP, Zheng JJ, et al. Efficacy and safety of mineralocorticoid receptor antagonists for patients with heart failure and diabetes mellitus: a systematic review and meta-analysis. BMC Cardiovasc Disord. 2016;16:28.

23 Cooper LB, Lippmann SJ, Greiner MA, Sharma A, Kelly JP, Fonarow GC, et al. Use of mineralocorticoid receptor antagonists in patients with heart failure and comorbid diabetes mellitus or chronic kidney disease. J Am Heart Assoc. 2017;6(12):e006540. 